\title{
Características de los usuarios del Centro de Recursos para el Aprendizaje y la Investigación de la Universidad de Cienfuegos
}

\author{
Aylén Pérez Borges \\ Amarilys Suárez Alfonso
}

Universidad de Cienfuegos "Carlos Rafael Rodríguez" - UCF, Cuba

\section{CASE REPORT}

\begin{abstract}
Resumo
Objetivo: Caracterizar a los usuarios del Centro de Recursos para el Aprendizaje y la Investigación (CRAI) de la Universidad de Cienfuegos "Carlos Rafael Rodríguez". Método: Se realizó un análisis bibliográfico de los términos relacionados con el estudio, un análisis de la metodología AMIGA, así como de las estadísticas que brinda el sistema automatizado de préstamos de esta biblioteca universitaria sobre los usuarios que más visitan el centro. Resultados: Se identificaron diversos grupos de usuarios a nivel de estudiantes, profesores, facultades, centros universitarios municipales, y centros de estudios; a la vez que dos grupos centrales: usuarios reales y potenciales. Conclusiones: Se conocieron algunas de las características de los usuarios del CRAI, partiendo de los grupos identificados por el centro, teniendo en cuenta aspectos como sus niveles educacionales e intereses. Se considera que un estudio más detallado puede quedar abierto para cumplimentar este objetivo a un nivel mayormente exhaustivo.
\end{abstract}

\section{Palavras-chave}

Centros de Recursos para el Aprendizaje y la Investigación ; Usuarios de la información ; Bibliotecas universitarias; Estudios de casos ; Universidad de Cienfuegos "Carlos Rafael Rodríguez" ; Cuba

\section{Characteristics of users of the Learning Resource Centre at the University of Cienfuegos}

\begin{abstract}
Objectives: Identify the characteristics of users of the Learning Resource Center at the University of Cienfuegos " Carlos Rafael Rodríguez ". Methods: It was developed an analysis of the terms related to the study, an analysis with AMIGA methodology, and an analysis of the university library statistics about users who visit the center. Results: The study identified severals user groups at the level of students, teachers, and research centers; while two main groups was identified: real users and potential users. Conclusions: The study identified the main characteristics of users of CRAI, but further study may help identify important aspects.
\end{abstract}

\section{Keywords}

Learning Resource Centers ; Information users ; University libraries ; Cases studies ; Universidad de Cienfuegos "Carlos Rafael Rodríguez"; Cuba 


\section{Introducción}

Los estudios de usuarios de información surgen en la década de los 20 del siglo XX. Hoy día es muy común encontrar estudios de este tipo en cualquier organización, aunque todos poseen diferentes niveles de profundidad, pero en esencia recogen datos e información general acerca de sus usuarios/clientes porque de ellos depende, en gran medida, su éxito. Los centros de información tienen la misión de satisfacer las necesidades de sus usuarios y es por esto que resulta imprescindible conocer sus características e intereses.

Conociendo las características de los usuarios, los diferentes centros de información pueden dar una atención más personalizada que les permita a estos, sentirse satisfechos y en confianza ante el especialista que lo ha ayudado a suplir su necesidad informativa, para no ignorar estas particularidades físicas, socioculturales y humanas en general, el sistema crea diferentes filtros o vías como son por ejemplo el registro de usuarios, buzón de quejas y sugerencias, boletas de solicitud con datos personales, estudios más profundos de la comunidad y mercado afines a la institución y sus principales lagunas, talleres de formación al personal que hace uso de sus productos y servicios, sistemas de referencia personales y online, así como otras ofertas de comunicación, según las características de las necesidades y sus respectivas formas de control y evaluación, experimentación del uso de técnicas de trabajo en grupo, solución de problemas y desarrollo de la creatividad en las ofertas de comunicación.

\section{Métodos y técnicas}

Para los estudios de usuarios se pueden utilizar metodologías, AMIGA, por citar una de ellas, se diferencia a otras en que prioriza el lugar de las personas y grupos humanos (formales e informales) de cualquier organización de información y su entorno, conocimientos, habilidades, hábitos, motivación, valores, inteligencia cognoscitiva y emocional, compatibilidad y otras características socio-psicológicas. Las personas se consideran y se registran, en sus diferentes funciones: como recursos -de conocimiento y aprendizaje en la organización, de carácter intangible, como usuarios con necesidades, como actores en los procesos de transferencia, comunicación y liderazgo en la organización, y se les otorga mayor peso y atención que a los recursos tangibles. Esta metodología se tuvo en cuenta para realizar un estudio que se propuso conocer las características de los usuarios, enfocado a un centro en particular: El CRAI de la Universidad de Cienfuegos, por lo que se plantea en este artículo el resultado de esa investigación, cuyo objetivo es: caracterizar los principales usuarios de esa institución.

Si se quiere hacer un estudio profundo de usuarios, es necesario centrarse en las personas más que en el sistema mismo, aunque el presente se considera un modesto aporte, resultado de las funciones de los especialistas de la información del área estudiada, como sistema que promueve el aprendizaje y la investigación en el contexto universitario.

\subsection{Actividades generales del CRAI}

La misión del CRAI de la Universidad de Cienfuegos es facilitar el acceso, la difusión y recuperación de los recursos de información, así como diseñar y producir materiales didácticos para apoyar el aprendizaje de los estudiantes. Adquirir, almacenar y crear nuevos espacios para la instrucción, con el fin de satisfacer las necesidades académicas de la universidad.

El CRAI tiene como visión apoyar la docencia y la investigación en la universidad "Carlos Rafael Rodríguez" logrando la convergencia entre los servicios tradicionales de biblioteca y los nuevos servicios que propician la tecnología de la información y las comunicaciones (TICs). Facilitando soporte al aprendizaje, poniendo a disposición de los estudiantes documentos pertinentes para su desarrollo educativo, ofreciéndoles asesoramiento, formando 
usuarios autosuficientes y proporcionándoles un entorno que favorezca su aprendizaje, con espacios para trabajos en grupo y con servicios complementarios.

Desde hace varios cursos, el CRAI viene realizando talleres enfocados en sus usuarios, para el mejor uso de sus productos y servicios, así como interpretación de herramientas tecnológicas por parte de estudiantes y profesores para la calidad de sus trabajos científicos y el sello bibliográfico que se debe fomentar en cada escrito académico y de rigor científico que formará parte del patrimonio universitario.

Las políticas editoriales de la entidad se han registrado y promocionado para apoyar el trabajo de técnicos y especialistas, las vías de comunicación se han establecido de manera variada (intranet, sitio de la biblioteca virtual, instalación de programas informáticos en facultades, sedes universitarias y centros de estudio, laboratorio, talleres de gestión de contenidos, cursos introductorios y de familiarización con el centro, visita por las diferentes salas, servicios personalizados).

Cada trabajador por su parte está encargado de asesorar en los aspectos científico-técnicos a un grupo determinado de usuarios, entre otras tareas, está la formación constante, mediante talleres, las búsquedas por temáticas en sitios referenciados y de prestigio internacional, participación en consejos de carrera, además de los procesos técnicos en cada sala de préstamo.

Es interés de la institución conocer las características de su comunidad usuario, a lo que pretende contribuir este artículo, no obstante un estudio más detallado quedaría abierto para complementar el trabajo.

\subsection{Términos relacionados con el estudio}

Es importante ubicarse en el espacio donde se realiza el estudio y sus características, o sea, el contexto actual en que se mueve. El CRAI de la Universidad de Cienfuegos es un centro sin fines de lucro, lo que marca la diferencia en la forma de denominar a sus usuarios. Un usuario es denominado cliente en determinadas circunstancias.

Según el Glosario de términos bibliotecológicos y de Ciencias de la Información de INFOMED, estos dos conceptos poseen más de una categoría, aunque de manera general la denominación usuario resulta más abarcadora, la que a su vez se divide en los siguientes tipos:

Usuario de la información: Persona, grupo o entidad, que utiliza la información o los servicios de información. Al estar involucrados los "usuarios" y trabajadores de la información en un proceso continuo de comunicación, el término se refiere a todos los que utilizan la información, sean o no trabajadores de la información.

Usuario potencial: Persona, grupo o entidad, cuya actividad está vinculada, directa o indirectamente, al cumplimiento de la misión y de los objetivos estratégicos de la organización o comunidad en la cual está inserta la entidad de información. Por ello, son el punto de partida para el cálculo de los recursos de información que se requieren (humanos, materiales, tecnológicos, financieros, organizacionales, así como la propia información.

Usuario real: Aquel usuario con el cual la entidad de información ya ha logrado establecer la comunicación. El Usuario potencial que aún no es real (al cual algunos autores llaman No Usuario), es aquel con el cual la entidad de información aún no ha logrado establecer la comunicación. El concepto se refiere a la información proveniente del servicio y de una entidad de información en particular, pues se sabe que todas las actividades humanas requieren información y por tanto, el usuario debe haber usado información de otras fuentes, incluso, posiblemente, la proveniente de servicios de otras entidades de información.

Cliente: Usuario que debe pagar directamente por los servicios/productos de la entidad de información, o sea, cuando media una relación monetario-mercantil directa entre ambas partes.

Cliente actual: Equivalente a Usuario Real, pero cuando media una relación monetario mercantil directa.

Cliente potencial: Aquel que es objeto de una estrategia para la venta de un producto/servicio por la entidad de información y que se toma como punto de partida para el cálculo de los recursos que la 
organización debe poner a disposición del mercadeo. Puede ser actual o haber prescindido ya de los servicios informacionales.

Cliente interno: Persona, grupo o entidad dentro de la organización que se toma como punto de referencia para el análisis de sistema. Cuando el autor citado utiliza este término siempre es referido al contexto del análisis interno del sistema que constituye la entidad de información, suponiendo que lo lógico es que no existan relaciones monetario-mercantiles directas entre cliente y proveedor, por cuanto pertenecen a la misma entidad, su funcionamiento es mutuamente necesario y, en última instancia, financiado a partir de los mismos fondos. En el caso de una entidad de información, son los propios trabajadores de la información y sus agrupaciones.

Cliente externo: Se refiere a la persona, grupo o entidad que no está subordinada ni administrativa, ni metodológicamente a la entidad de información o que posee una institución intermedia para los servicios de información. Sería utilizado siempre en el context del análisis de sistema e independientemente de si se establecen o no relaciones monetario - mercantiles directas y de si son actuales o potenciales.

De manera generasl, todos estos tipos presentan necesidades relacionadas con la entidad. Para hacer un estudio de usuarios o necesidades, por muy sencillo que este sea, es necesario el análisis de los diferentes criterios que indica la literatura y una visión amplia de lo implicado en el proceso, así la fuente puede servir a cualquier investigador.

No deben obviarse algunos requisitos importantes como: establecer una tipología de usuarios, identificar sus necesidades atendiendo a las temáticas que ellos más consultan y temas de investigación, conocer además su profesión y nivel educacional.

\subsection{Categorías generales para el estudio}

Cada entidad de información debe determinar la cantidad y variedad de categorías de usuarios que le corresponden o se relacionan de cierto modo con la misma, estas categorías pueden aparecer registradas en el libro de inscripción y boletas de solicitud o bien elaborarse un mapa de recursos de información, conocimiento o aprendizaje.

EI CRAI de la Universidad de Cienfuegos cuenta con un sistema automatizado de préstamo, donde incluye algunas de las características de sus usuarios, y como requisito para gestionar cada préstamo la persona que hace la solicitud debe presentar su carnet de identidad como confirmación de que sus datos personales han quedado registrados antes.

Dentro de la tipología usuario incluye las categorías de: estudiante, profesor, investigador y otros, lo que da una medida de los principales consumidores de información del centro.

La metodología AMIGA se centra en tres categorías principales para el estudio de usuarios a los que denomina círculos concéntricos:

- Trabajadores de las entidades de información.

- Resto de las personas y grupos dentro de la organización.

- Aquellos que pertenecen al entorno y desempeñan papeles de colaboradores, reguladores, suministradores, distribuidores, clientes, competidores y otros relacionados con la organización.

Esta metodología, la que está muy bien concebida y se puede utilizar parcialmente en la organización que realiza el estudio de usuarios y necesidades, plantea que se debe disponer de un mapa y registro del lugar prioritario de las personas y grupos humanos (formales o informales) de la organización y de su entorno, sus conocimientos, habilidades, hábitos, motivación, valores, inteligencia cognoscitiva y emocional, compatibilidad y otras características socio-psicológicas, lo que se puede estudiar en una investigación más amplia. 
Los aspectos teóricos evidencian que el tema de los usuarios va más allá de los límites de la institución que realiza la investigación, se sale de sus marcos, y que un estudio de este tipo conlleva tiempo y técnica. Generalmente los instrumentos son muy variados, se deben realizar encuestas, entrevistas, técnicas grupales, independientemente del análisis bibliográfico que corresponde al investigador.

Es importante ser específico a la hora de establecer las diferentes categorías para el estudio de usuarios, necesidades o características principales. El sistema automatizado del CRAI comenzó a ponerse en práctica durante el curso 2009-2010, y aún necesita incluir datos para la gestión de información. Se han detectado deficiencias desde su funcionamiento, y poco a poco se han ido incluyendo carreras omitidas en un inicio, y categorías no registradas, pero ha logrado registrar información detallada por grupo, más adelante se brindarán algunas estadísticas que produjo este registro en el sistema. No obstante, pueden crearse otros productos que indiquen las características de usuarios de forma más detallada y ramificada.

\section{Resultados}

\subsection{Grupos y características de los usuarios del CRAI de la Universidad de Cienfuegos.}

En la práctica, muchos grupos, además de los que se incluyen en el sistema automatizado del CRAI, poseen características, intereses o niveles educacionales específicos.

\section{Estudiantes}

Primer grupo del sistema, se diferencian entre sí por intereses y niveles. Pueden determinarse, por tanto, varias categorías dentro de este grupo:

- Estudiantes de grado: Muchas veces desconocen las fuentes que desean utilizar, necesitan una formación científica y metodológica continua, han comenzado sus primeros pasos camino a la investigación. Necesitan vincular sus investigaciones con la práctica que ejercerán y obtener habilidades. Utilizan en la mayoría de los casos publicaciones seriadas, libros, información digital y todo lo que puedan recolectar que sea pertinente a su estudio, pero no son capaces de investigar un tema por si solos, porque en la práctica demuestran una dependencia del profesor que le indica concretamente lo que necesitan para la asignatura.

- Estudiantes de postgrado: Están en constante superación, necesitan información actualizada, tienen mayor experiencia y sus necesidades están a un nivel más elevado, poseen una necesidad urgente de adquirir habilidades asociadas con la práctica de su profesión. Utilizan manuales, artículos, documentos electrónicos más específicos.

- Estudiantes de maestría y doctorado: Necesitan búsquedas de fuentes referenciadas y más exhaustivas, incluso en otros idiomas. Son personas maduras y están a un nivel cultural más elevado. Las fuentes que consultan son por lo general trabajos de diploma, bases de datos, publicaciones periódicas, revistas de impacto.

- Estudiantes extranjeros (de preparatoria): No dominan el idioma, necesitan orientarse y apoyarse en fuentes que logren comprender, sus preguntas son sencillas, usan documentos relativos a la gramática y el idioma en general (diccionarios).

Cada uno de estos cuatro tipos posee sus características específicas y los intereses no son los mismos, aún cuando su misión es común, porque todos ellos persiguen aprender y finalmente graduarse. Si se hace 
un análisis general, todos los usuarios de información necesitan obtener la información o saber cómo llegar a ella, pero al final las vías de recuperación pueden variar en cada caso. El especialista debe tener en cuenta estas características, además de ubicarse en contexto, que es un elemento primordial para la satisfacción de la persona que hace la solicitud.

\section{Profesores}

Dentro del segundo grupo existen varios tipos con sus especificidades:

- Profesor dedicado de a lleno a la enseñanza: Esta categoría tiene una especificidad, y es que quizá el profesor que se dedica a tiempo completo a enseñar no está en contacto con los últimos adelantos de la recuperación de información, ya sea por falta de tiempo, porque no presenta una continua formación o no posee los recursos necesarios, lo que puede ser relativo. Utiliza por lo general bibliografía básica, además de artículos científicos e información digital.

- Profesor que trabaja como tecnólogo o científico: Está más actualizado de lo que pasa en el mundo de la información, dispone de mayor tiempo para su preparación, debe tener mayor cultura infotecnológica y contar con recursos más potentes. Es necesario registrar su localización, porque este puede estar más distante y necesitar una mayor constancia del profesional de información para el préstamo y recibo de documentos. Sus necesidades varían mucho, pueden estar a la par de las tecnologías y políticas científicas. Utilizan programas informáticos, internet, publicaciones periódicas, revistas.

También podrían agruparse según sus categorías docentes, que ubican al profesor a un nivel menor o mayor, si se valora que teniendo en cuenta la categoría, así será su sistema de conocimientos, preparación, experiencia, etc.

Los profesores, a diferencia de los estudiantes conocen y saben dirigirse a las fuentes que necesitan.

Asimismo, estos profesores, al igual que lo estudiantes, corresponden a distintos lugares, universidades municipales, facultades, centros de estudios, carreras, disciplinas, etc., dentro del centro y la provincia, aunque todos los que se relacionan con la Educación Superior en Cuba, principalmente estudiantes, profesores e investigadores de otras universidades, pueden considerarse usuarios del centro de información porque pueden necesitar hacer uso de sus productos y/o servicios directa o indirectamente.

\section{Facultades}

- Facultad de Ciencias Sociales y Humanísticas

Historia

Socioculturales

Inglés

Derecho

- Facultad de Ingeniería

Informática

Mecánica

Química

- Facultad de Agronomía Agronomía 
- Facultad de Ciencias Económicas y Empresariales

Economía

Contabilidad

Industrial

- Facultad de Cultura Física

Cultura física

Cada una de las facultades tiene intereses relacionados con las distintas carreras y temas afines, por lo que requieren, en la mayoría de los casos, información especializada. Para cada grupo se han realizado búsquedas de artículos en bases de datos específicas y sitios científicos a los que pueden acceder, los que se encuentran registrados en la biblioteca virtual de la Universidad de Cienfuegos. En el caso de profesores e investigadores, pueden asociarse con esos sitios para publicar sus trabajos, incluyendo revistas referenciadas y de impacto en bases de datos online y Web de la Ciencia. Se comprobaron además todos los URL que entregaron los técnicos y especialistas encargados de recoger la información en las distintas facultades y centros de estudio sobre sus principales líneas de investigación, para no provocar deficiencias en el acceso y la recuperación documental.

Se concibieron en los gestores de referencias bibliográficas orientados para trabajos científicos de los graduados e investigadores, normas relacionadas con las diferentes ciencias para permitir la satisfacción y el ajuste a sus necesidades.

Se impartieron talleres que permitieron una relación con los recursos y servicios del CRAI y las diversas especialidades.

\section{Centros Universitarios Municipales (CUM)}

- Aguada

- Abreu

- Rodas

- Sede Central(Cienfuegos)

- Palmira

- Cruces

- Lajas

Estos son usuarios que deben tener un seguimiento por parte del centro, cada especialista y técnico de servicio del CRAI tiene a cargo uno o varios de los centros municipales según su compatibilidad, y realizan visitas a los municipios, planifican talleres cuando visitan la entidad, por lo que están en comunicación constante con los profesores que hacen uso de los servicios de información, ya sea de forma personalizada o por vías como el email.

La mayoría de los usuarios que radican en los municipios presentan problemas para conocer la información referente a las actividades y orientaciones que emite el centro de información, pero se han creado las vías para que puedan tener acceso a ella.

La biblioteca virtual disponible dentro de la Universidad en: http://biblioteca.ucf.edu.cu/, se ha visitado desde diferentes sedes para comprobar su conectividad a la sede central y se mantiene actualizada en los recursos que se innovan y orientaciones emitidas para la armonía del sector informacional en el contexto 
local. Actualmente todos estos centros alejados de la sede central universitaria han sido visitados al menos una vez por un miembro del CRAI y han recibido talleres de gestión de contenidos. El municipio de Abreu según informó la especialista principal es el que más problemas presenta porque el acceso a través de la transportación se ve afectado.

Se ha demostrado una mayor flexibilidad con los profesores de este municipio cuando acuden al CRAI por cualquier motivo, y siempre reciben una orientación y trato personalizado que está en dependencia de esta peculiaridad de la localización para que los mismos puedan cumplimentar su misión a pesar de su fatalidad geográfica.

\section{Centros de Estudio}

- Centro de Estudio de la Didáctica y Dirección de la Educación Superior(CEDDES)

- Centro de Estudio de Energía y Medio Ambiente(CEEMA)

- Grupo de Estudios de Gerencia Organizacional (GEGO)

- Centro de Estudios para la Transformación Agraria Sostenible(CETAS)

Los centros de estudios reciben atención por parte del personal del CRAl, al igual que los estudiantes y profesores de las facultades, están conformados por prestigiosos investigadores del centro que participan en talleres de gestión de contenido y reciben orientaciones para que sus publicaciones estén acorde con los requisitos de la editorial universitaria, que se incluye dentro del CRAI. Pueden considerarse investigadores en su gran totalidad, independientemente de que incluyan a los demás grupos como profesor y algunos adiestrados que recién se han gradudado de su especialidad.

\section{Científicos, tecnólogos e investigadores:}

Esta categoría muestra especial interés en la búsqueda del conocimiento, en la creación de ideas y el descubrimiento de nuevas relaciones y su aplicación. Buscan satisfacer sus necesidades materiales.

En la categoría "otros" cabrían los técnicos, especialistas, profesionales y trabajadores de la universidad en general, que tienen una orientación por cumplir, y sus actividades están basadas en los motivos de búsqueda y en la naturaleza de su trabajo. Orientación altamente práctica, intereses por resolver problemas. Necesitan incrementar su contribución social.

\subsection{Clasificación de los usuarios del CRAI}

Teniendo en cuenta los términos abordados, las categorías expuestas(o tipos para cada grupo) y las características de los mismos, los usuarios de información del centro estudiado se pueden clasificar en:

- Usuarios reales: Estudiantes, Profesores e Investigadores de la Universidad de Cienfuegos y de los Centros Universitarios Municipales, incluyendo además los propios trabajadores del CRAI y de la universidad en general, que han establecido contacto con sus especialistas o viceversa, los que hacen uso real de sus productos y servicios. Cada especialista y técnico del CRAl tiene a su cargo un grupo determinado de usuarios y es su tarea hacer que la información llegue a ellos, ya sea dentro de la ciudad o en un 
municipio específico. Deben promocionar sus recursos de información y hacer que los usuarios que están en contacto directa o indirectamente con ella hagan uso eficiente de las fuentes bibliográficas y tecnologías, entre ellos programas informáticos que colaboran a la eficiencia en la investigación y el trabajo científico y académico, por tanto, cada uno de los centros de estudios identificados, los centros universitarios municipales, facultades y carreras deben tener una noción de lo que hace el CRAI. Los usuarios que más visitan el CRAI son los reales y también los que se han sido concebidos para brindar sus servicios, pueden incluirse además los suministradores y personal del entorno que se encuentra comunicándose con el centro, directa o indirectamente.

- Usuarios potenciales: Otros usuarios que no se relacionan directa o indirectamente con el centro, pero que existen y pueden hacer uso de sus recursos, incluyendo estudiantes y profesores de organizaciones de información, centros de estudios de la Educación Superior en el país. Pueden ubicarse aquí algunos competidores por ejemplo, que no hacen uso de la información pero que de cierto modo existen y necesitan tomarse en cuenta para diseñar productos informacionales y brindar un servicio de información.

\section{Discusión}

Las estadísticas del sistema automatizado (SIB) del CRAI, las boletas de solicitud de las diferente áreas de servicio y el libro de registro, indican que los usuarios que más visitan el centro son estudiantes, dentro de este grupo se desglosan los que pertenecen a las distintas carreras y la mayor cantidad responde a las carreras de Estudios Socioculturales e Ingeniería Industrial.

Algunas de las informaciones que se emiten, responden a planteamientos y debates en los consejos de carrera de estas facultades.

El primer grupo (estudiantes de Estudios Socioculturales), la mayoría de las veces consulta documentos de la sala de Ciencias Sociales (Humanidades) y en algunos casos obras de referencia pertinentes a estas ciencias. También hacen uso de la Hemeroteca, deben utilizar Revistas como Temas, Catauro y la Gaceta de Cuba que les orientan en clase según los planteamientos de varios profesores del departamento.

Es muy difícil afirmar con toda certeza que de estos resultados dependan sus características socio-psicológicas (conocimientos, habilidades, hábitos, motivación, valores, inteligencia cognoscitiva y emocional, etc.), pero por lo general tienden más a investigar que otros estudiantes que se dedican a las ciencias técnicas, pues estos últimos van más a lo concreto y la aplicación práctica de las cosas.

Podría afirmarse que de cierta manera los lectores más sobresalientes poseen un mayor sentido de pertenencia y responsabilidad, además de que tienden a ser futuros investigadores mejor preparados en el uso de herramientas metodológicas y bibliográficas.

Los estudiantes de Estudios Socioculturales consultan bibliografía de corte cultural, literario, artístico, lo que los ubica a un conocimiento y elevación más directa con la literatura en general.

La sala de Ciencias Técnicas del centro de información (CRAI) es mayormente visitada por estudiantes de la carrera de Ingeniería industrial que utilizan materia concreta de su especialidad (libros, manuales, tablas) y no tienen noción de publicaciones seriadas de interés, según los estudios de usuario realizados en esta área.

\section{Conclusiones}

Se conocieron algunas de las características de los usuarios del CRAI, partiendo de los grupos identificados por el centro, teniendo en cuenta aspectos como sus niveles educacionales e intereses. 
Una investigación más detallada puede quedar abierta para caracterizar los principales usuarios de la institución estudiada a un nivel mayormente exhaustivo. Un estudio de usuarios de la información de cualquier entidad conlleva tiempo y puede parecer muy subjetivo, se necesitan aplicar técnicas variadas como encuestas, tormentas de idea, sicométricos, entre otras que permitan estimar cómo son los usuarios realmente y para que sea más efectiva su proyección social.

\section{Bibliografía}

Fernández, M de las M y otros. (2007). Metodología para el estudio de las necesidades de información, conocimiento y aprendizaje en las bibliotecas y centros de documentación de salud. Dialnet. 15 (4). Disponible en:

http://dialnet.unirioja.es/servlet/articulo?codigo=2267112

Glosario de términos bibliotecológicos y de Ciencias de la Información. (2009). Infomed: Biblioteca Médica Nacional. Disponible en: http://www.sld.cu/sitios/bmn/temas. php?idv=3015.

González, C. (2006). Usuarios de la información: formación y desafíos. En: Biblioteca Médica Nacional de Cuba. Investigación de Mercados: un enfoque aplicado. Tercera edición.

Núñez, I. (2005). AMIGA: una metodología integral para la determinación y la satisfacción dinámica de las necesidades de formación e información en las organizaciones y comunidades. En: Base de dados LILACS. Disponible en:

http://www.bvs.sld.cu/revistas/aci/vol12 4 04/aci02404.htm.

Pojuán, G. (2007). Sistemas de Información: Principios y aplicaciones. La Habana: Editorial Félix Varela.

Santaella, R. D. (2005). Metodología de estudios de usuarios de información. Estudio de casos en la Administración Pública. Revista TEXTOS de la CiberSociedad. 5. Disponible en: http://www.cibersociedad. net.

Zamora, R. (2012). La evolución de biblioteca a Centros de Recursos para el Aprendizaje y la Investigación (CRAI). Revista Universidad y Sociedad. 4(1), enero/abril. Disponible en: http://rus.ucf.edu.cu/index.php/UyS/article/view/377. 
BIUlí Características de los usuarios del Centro de Recursos para el Aprendizaje

\section{Datos de las autoras}

Aylén Pérez Borges

Especialista en Análisis, Procesamiento y Servicios de la Información. CRAI. Universidad de Cienfuegos.

aborges@ucf.edu.cu

Amarilys Suárez Alfonso

Especialista en Análisis, Procesamiento y Servicios de la Información. CRAI. Universidad de Cienfuegos.

asuarez@ucf.edu.cu

Recibido-Received : :2013-11-28

Aceptado-Accepted : : $2013-12-30$

\section{(c) EY}

This work is licensed under a Creative Commons Attribution 4.0 United States License.

\section{ULLIS $\underline{D}$ - Sonk}

This journal is published by the University Library System of the University of Pittsburgh as part of its $\underline{D}$-Scribe Digital Publishing Program and is cosponsored by the University of Pittsburgh Press. 